\title{
Locally Restricted Compositions IV. Nearly Free Large Parts and Gap-Freeness
}

\author{
Edward A. Bender ${ }^{1}$, E. Rodney Canfield $\|^{\|}$and Zhicheng Gao $\|^{3}$ \\ ${ }^{1}$ Department of Mathematics, University of California at San Diego, La Jolla, CA, USA \\ ${ }^{2}$ Department of Computer Science, University of Georgia, Athens, GA, USA \\ ${ }^{3}$ Department of Mathematics and Statistics, Carleton University, Ottawa, Ontario, Canada
}

We define the notion of $t$-free for locally restricted compositions, which means roughly that if such a composition contains a part $c_{i}$ and nearby parts are at least $t$ smaller, then $c_{i}$ can be replaced by any larger part. Two wellknown examples are Carlitz and alternating compositions. We show that large parts have asymptotically geometric distributions. This leads to asymptotically independent Poisson variables for numbers of various large parts. Based on this we obtain asymptotic formulas for the probability of being gap free and for the expected values of the largest part and number distinct parts, all accurate to $o(1)$.

Keywords: restricted compositions, largest part, distinct parts, gaps

\section{Introduction}

Locally restricted compositions (defined in [2]) are a broad generalization of ordinary compositions that include Carlitz and up-down compositions as well as various lattice paths counted by area. A recurrence condition rules out partitions. Generating functions were computed for special cases of locally restricted compositions in [1] and [3]. Although it was not possible to compute generating functions in the general case, various properties were established, including the following.

(a) The number of compositions of $n$ is $A r^{-n}\left(1+O\left(\delta^{n}\right)\right)$ for some $0<\delta<1$ because of a simple pole in the generating function. Since the convergence to $A r^{-n}$ is exponentially fast, the value of $r$ can be estimated fairly easily if one can count compositions for relatively small values of $n$. [2, Theorem 3]

(b) If a subcomposition can occur arbitrarily often, the number of times it occurs in a random composition of $n$ has a distribution that is asymptotically normal with mean and variance asymptotically proportional to $n$. The same is true for the total number of parts in a random composition. [2, Theorem 4]

\footnotetext{
${ }^{\dagger}$ Research supported by NSA Mathematical Sciences Program.

$\ddagger$ Research supported by NSERC.

1365-8050 @ 2012 Discrete Mathematics and Theoretical Computer Science (DMTCS), Nancy, France
} 
(c) In many cases, the largest part and number of distinct parts in a random composition is asymptotic to $\log _{1 / r} n$. [2, Section 9]

In none of these papers was the behavior of the large parts addressed beyond that in (c).

Here we study large part behavior, which is closely related to other properties such as number of distinct parts and the probability of being gap free. The basic idea is to define a subclass of locally restricted compositions for which we can show that the large parts are asymptotically independent geometric random variables. This leads to asymptotically independent Poisson random variables for numbers of various large parts. Our main goal is to prove Theorem 1. A full understanding of it requires some definitions.

Definition $1 \mathbb{N}$ and $\mathbb{N}_{0}$ denote the positive integers and the positive integers and 0 , respectively.

A composition is written $\overrightarrow{\mathbf{c}}=c_{1} \cdots c_{k}$ where $c_{i} \in \mathbb{N}$. (We never write it as $c_{1} \ldots c_{k}$.) We use the same notation to denote concatenation of compositions as in $\overrightarrow{\mathbf{a}}_{1} \cdots \overrightarrow{\mathbf{a}}_{m}$. A subcomposition of $\overrightarrow{\mathbf{c}}$ is a sequence of one or more consecutive parts of $\overrightarrow{\mathbf{c}}$.

The length of $\overrightarrow{\mathbf{c}}=c_{1} \cdots c_{k}$ is denoted by $\operatorname{len}(\overrightarrow{\mathbf{c}})=k$ and the sum of the parts by $\Sigma(\overrightarrow{\mathbf{c}})$.

Definition 2 (Local restriction function) Let $m, p \in \mathbb{N}$. A local restriction function of type $(m, p)$ is a function

$$
\Phi:\{0,1, \ldots, m-1\} \times\left(\mathbb{N}_{0}\right)^{p+1} \rightarrow\{0,1\}
$$

with $\Phi(i ; 0, \ldots, 0)=1$ for all $i$. The integers $m$ and $p$ are called, respectively, the modulus and span of $\Phi$.

Definition 3 (Class of compositions determined by $\Phi$ ) Let $\Phi$ be a local restriction function. The class of compositions determined by $\Phi$ is

$$
\mathcal{C}_{\Phi}=\left\{\overrightarrow{\mathbf{c}}: \overrightarrow{\mathbf{c}} \text { is a composition, and } \Phi\left(i \bmod m ; c_{i}, c_{i-1}, \ldots, c_{i-p}\right)=1 \text { for } i \in \mathbb{Z}\right\} .
$$

If an index $j$ refers to a part before the first part $(j<1)$ or after the last part $(j$ greater than the number of parts), we set $c_{j}=0$.

A class $\mathcal{C}$ of compositions is locally restricted if $\mathcal{C}=\mathcal{C}_{\Phi}$ for some local restriction function $\Phi$.

The number $m$ determines a periodicity. The number $p$ determines a "window"-by looking at parts $c_{j}$ with $0<|i-j| \leq p$, we can determine what values, if any, are allowed for $c_{i}$. We could replace $m$ by any multiple of itself, $p$ by any larger value, and redefine $\Phi$ to get the same class of compositions.

Example 1 (Alternating compositions) The up-down compositions $c_{1}<c_{2}>c_{3}<\cdots$ can be described as follows. We set $m=2$ and $p=1$. When $0<a<b$, define $\Phi$ as follows:

$$
\Phi(1 ; a, 0)=1, \quad \Phi(1 ; a, b)=1, \quad \Phi(0 ; b, a)=1, \quad \Phi(0 ; 0,0)=1, \quad \Phi(1 ; 0,0)=1,
$$

and $\Phi=0$ otherwise.

Definition 4 (Recurrent compositions) Let $\mathcal{C}$ be a class of locally restricted compositions with span $p$ and modulus $m$.

We say that a subcomposition $\overrightarrow{\mathbf{s}}$ is recurrent at $j$ modulo $m$ (often shortened to recurrent) if, for every $k$ and every $\overrightarrow{\mathbf{a}} \overrightarrow{\mathbf{x}} \overrightarrow{\mathbf{z}} \in \mathcal{C}$ with $\operatorname{len}(\overrightarrow{\mathbf{a}}) \geq p$ and $\operatorname{len}(\overrightarrow{\mathbf{z}}) \geq p$, there is a composition $\overrightarrow{\mathbf{a}} \cdots \overrightarrow{\mathbf{z}} \in \mathcal{C}$ containing at least $k$ copies of $\overrightarrow{\mathbf{s}}$ starting at positions congruent to $j$ modulo $m$. If a recurrent subcomposition has length 1 , we call it a recurrent part.

A class $\mathcal{C}$ is recurrent if the following hold. 
- Every subcomposition $c_{i} \cdots c_{j}$ of $\overrightarrow{\mathbf{c}} \in \mathcal{C}$ with $i>p$ and $j+p \leq \operatorname{len}(\overrightarrow{\mathbf{c}})$ is recurrent.

- If $\overrightarrow{\mathbf{r}}$ and $\overrightarrow{\mathbf{s}}$ are recurrent subcompositions and $\overrightarrow{\mathbf{a}} \overrightarrow{\mathbf{x}} \overrightarrow{\mathbf{z}} \in \mathcal{C}$, then there is a composition $\overrightarrow{\mathbf{a}} \cdots \overrightarrow{\mathbf{r}} \cdots \overrightarrow{\mathbf{s}} \cdots \overrightarrow{\mathbf{z}}$ in $\mathcal{C}$.

In Example 1 the subcomposition $a b$ is recurrent at 1 modulo 2 whenever $0<a<b$ and is recurrent at 0 modulo 2 whenever $0<b<a$. The part 1 is recurrent at 1 modulo 2 but is not recurrent at 0 modulo 2 .

Remark 1 (Ignoring nonrecurrent parts) Since nonrecurrent parts can only appear in the first or last p parts, and since almost all compositions of $n$ have $\Theta(n)$ parts, we can usually ignore the nonrecurrent parts in our asymptotic estimates.

Definition 5 ( $t$-free) Let $\mathcal{C}$ be a class of recurrent compositions. If the following hold, we say that $\mathcal{C}$ is $t$-free.

(a) There is a recurrent subcomposition $\overrightarrow{\mathbf{r}}=r_{1} \cdots r_{p} x r_{p+2} \cdots r_{2 p+1}$ such that $x \geq t+\max \left(r_{1}, \ldots, r_{2 p+1}\right)$.

(b) If $\overrightarrow{\mathbf{r}}=r_{1} \cdots r_{p} x r_{p+2} \cdots r_{2 p+1}$ is a subcomposition of $\overrightarrow{\mathbf{c}} \in \mathcal{C}$ for some $r_{i}$ and some $x \geq t+\max \left(r_{1}, \ldots, r_{2 p+1}\right)$ and if $x^{\prime} \geq t+\max \left(r_{1}, \ldots, r_{2 p+1}\right)$, then replacing $x$ by $x^{\prime}$ in this subcomposition of $\overrightarrow{\mathbf{c}}$ gives a composition $\overrightarrow{\mathbf{c}}^{\prime} \in \mathcal{C}$.

It is fairly easy to verify that $t$-free $\mathcal{C}_{\Phi}$ are special cases of the regular $\mathcal{C}_{\Phi}$ studied in [2].

Example 2 (Generalized Carlitz compositions) Carlitz compositions are defined by the restriction $c_{i} \neq$ $c_{i-1}$. They were generalized to restricted differences in [1] by requiring that $c_{i}-c_{i-1} \notin \mathcal{N}$ where $\mathcal{N}$ is an arbitrary set of integers. (Carlitz compositions correspond to $\mathcal{N}=\{0\}$.) These compositions are recurrent with modulus 1 and span 1 . If $\mathcal{N}$ is finite, they are $t$-free compositions for all $t$ greater than the maximum of $|n|$ over $\mathcal{N}$.

Example 3 (Some periodic conditions) Up-down compositions have constraints of modulus 2. General periodic inequality constraints were studied in [3]. These are all 1-free provided they allow parts to both increase and decrease.

For fixed $k$, $k$-rowed compositions in which adjacent parts must differ are 1-free. One interleaves the parts to produce a one-rowed composition: If $a_{i, j}$ are the parts of a k-rowed composition of $n$, then $c_{i+k(j-1)}=a_{i, j}$ for $1 \leq i \leq k$ and $j=1,2, \cdots$ gives a bijection with one-rowed compositions $\overrightarrow{\mathbf{c}}$ of $n$. We can take the modulus and span to be $k$.

Definition 6 (Gap free) A composition with largest part $M$ is called gap free if it contains all recurrent parts less than $M$.

The restriction of gap-free to recurrent parts is used to rule out classes such as the following. Let $\mathcal{C}$ be all compositions subject to the restriction that 2 and 3 can appear only as the first part of a composition. Since almost all compositions contain 1 and no composition in $\mathcal{C}$ can contain both 2 and 3 , almost no compositions in $\mathcal{C}$ would be gap-free if we required that the support of the parts be an interval in $\mathbb{N}$.

Conventions 1 We use the following conventions in this paper. 
- When we talk about something random, we always mean that it is chosen uniformly at random from the set in question. We say that a property holds asymptotically almost surely (a.a.s) if the probability that the property holds tends to 1 as the size of the set goes to infinity, and we also say that almost all objects in the set have the property.

- Expectation is denoted by $\mathrm{E}$.

- After a class of compositions has been defined, we usually omit the modifiers (e.g. t-free) and refer to elements of the class simply as compositions.

- The number of compositions of $n$ in the class $\mathcal{C}$ is asymptotically $A r^{-n}$. We will always use $A$ and $r$ for these parameters. Note that $r$ is the radius of convergence of the generating function for class $\mathcal{C}$.

- All logarithms are to the base $1 / r$ except the natural logarithm $\ln$.

Theorem 1 (Main theorem) Let $r$ be the radius of convergence of the generating function of a class of $t$-free compositions. Let $\gamma \doteq 0.577216$ be Euler's constant and let

$$
P(x)=\frac{1}{\ln (1 / r)} \sum_{k \neq 0} \Gamma\left(\frac{2 i \pi k}{\ln (1 / r)}\right) \exp (-2 i k \pi \log x) .
$$

(For $1 / 2<r<1$ this is a periodic function of $\log x$ with amplitude less than $10^{-6}$.)

The following are true for some $C>0$, which has the same value in all parts of the theorem.

(a) Select a composition of $n$ uniformly at random. Let $X_{0}(n)$ be the number of parts and $X_{k}(n)$ the number of parts of size $k$. For recurrent $k$ and $\epsilon>0$,

$$
\operatorname{Prob}\left(\left|\frac{X_{k}(n)}{X_{0}(n)}-\frac{\mathrm{E}\left(X_{k}(n)\right)}{\mathrm{E}\left(X_{0}(n)\right)}\right|>\epsilon\right) \rightarrow 0 \text { as } n \rightarrow \infty .
$$

Furthermore, the limit

$$
u_{k}=\lim _{n \rightarrow \infty} \frac{\mathrm{E}\left(X_{k}(n)\right)}{\mathrm{E}\left(X_{0}(n)\right)}
$$

exists, and $u_{k} \sim B r^{k}$ as $k \rightarrow \infty$ for some positive constant $B$.

(b) Let the random variable $M_{n}$ be the size of the maximum part in a random composition of $n$. For any function $\omega_{b}(n)$ such that $\omega_{b}(n) \rightarrow \infty$ as $n \rightarrow \infty,\left|M_{n}-\log (n)\right|<\omega_{b}(n)$ a.a.s. Furthermore

$$
\mathrm{E}\left(M_{n}\right)=\log \left(\frac{C n}{1-r}\right)+\frac{\gamma}{\ln (1 / r)}-\frac{1}{2}+P\left(\frac{C n}{1-r}\right)+o(1)
$$

where $C=B \lim _{n \rightarrow \infty} \mathrm{E}\left(X_{0}(n)\right) / n$.

(c) Let $\nu$ be the number of nonrecurrent parts. (Since the compositions are $t$-free, $\nu$ is finite.) Let the random variable $D_{n}$ be the number of distinct recurrent parts in a random composition of $n$. For any function $\omega_{c}(n)$ such that $\omega_{c}(n) \rightarrow \infty$ as $n \rightarrow \infty,\left|D_{n}-\log (n)\right|<\omega_{c}(n)$ a.a.s. Furthermore

$$
\mathrm{E}\left(D_{n}\right)+\nu=\log (C n)+\frac{\gamma}{\ln (1 / r)}-\frac{1}{2}+P(C n)+o(1) .
$$


(d) Let $q_{n}(k)$ be the fraction of compositions of $n$ which are gap-free and have largest part $k$. There is a function $\omega_{d}(n) \rightarrow \infty$ as $n \rightarrow \infty$ such that

$$
q_{n}(k) \sim \exp \left(\frac{-C n r^{k+1}}{1-r}\right) \prod_{j \leq k}\left(1-\exp \left(-C n r^{j}\right)\right)
$$

uniformly for $|k-\log (n)|<\omega_{d}(n)$. Furthermore, for any constant $D$, the minimum of $q_{n}(k)$ over $|k-\log (n)|<D$ is bounded away from zero.

(e) Let $q_{n}$ be the fraction of compositions of $n$ which are gap-free. Then $q_{n}$ is asymptotic to the sum of the right side of (4), where the sum may be restricted to $|k-\log (n)|<\omega_{d}(n)$ for any $\omega_{d}(n) \rightarrow \infty$ as $n \rightarrow \infty$. Furthermore, $q_{n} \sim p_{m}$ where $m=\left\lfloor\frac{C n}{1-r}\right\rfloor$ and

$$
p_{m}= \begin{cases}1 & \text { if } m=0 \\
\sum_{k=0}^{m-1} p_{k}\left(\begin{array}{c}
m \\
k
\end{array}\right) r^{k}(1-r)^{m-k} & \text { if } m>0\end{cases}
$$

(f) Let $g_{n}(k)$ be the fraction of compositions of $n$ that have exactly $k$ parts of maximum size. Then for each fixed $k$ and as $n \rightarrow \infty$,

$$
g_{n}(k) \sim \frac{(1-r)^{k} \log e}{k !} \sum_{\ell=-\infty}^{\infty} \Gamma(k+2 \pi i \ell \log e) \exp \left(-2 \pi i \ell \log \frac{C n}{1-r}\right) .
$$

We recall that $\Gamma(a+i y)$ goes to zero exponentially fast as $y \rightarrow \pm \infty$. Thus sums in the theorem with complex gamma functions are dominated by the small index values.

Proofs are omitted, however intermediate results that we use in proving Theorem 1 are included.

\section{Discussion and Examples}

Remark 2 (Some previous results) The asymptotic probability that an unrestricted composition is gapfree was obtained by Hitczenko and Knopfmacher [8]. Various authors have considered the number of distinct parts in unrestricted compositions [10, 11]. Louchard [14] also studied the number of distinct parts of given multiplicity.

The number of distinct parts in Carlitz compositions were studied by Hitczenko and Louchard [9] who required an independence assumption that was eliminated by Goh and Hitczenko [7]. Knopfmacher and Prodinger [13] obtained asymptotics for the largest part in Carlitz compositions. The various results for Carlitz compositions were observed to have a small periodic oscillation.

Theorem 1 extends most of these results to t-free compositions. Whereas our error terms are o(1), some of the previous results have greater accuracy. Also, we do not have formulas for the two constants $C$ and $r$ appearing in our results; however, the more important $r$ is easy to estimate if one can count compositions efficiently. 
Example 4 (Gap-free) The numbers $p_{m}$ in (e) were studied by Hitczenko and Knopfmacher [8] who showed that they oscillated with the same period as (1) when $r>1 / 2$. They showed that, for $r=1 / 2$, there is no oscillation. Their Figure 7 shows that the amplitude of oscillation of $p_{m}$ is less than $10^{-6}$. Consequently, if $r$ is known, one can determine the asymptotic value of $p_{m}$ and hence $q_{n}$ to within $10^{-6}$. The following are the values of $p_{m}$ for three families of compositions, correct up to the sixth decimal place.

- For Carlitz compositions, it is known $r \doteq .57134979$. It follows from (5) that $p_{m} \doteq 0.372000$ for $m \geq 25$.

- For strictly alternating compositions $\left(c_{i-1}<c_{i}>c_{i+1}\right), r \doteq 0.63628175$ by [3]. It follows from (5) that $p_{m} \doteq 0.252277$ for $m \geq 25$.

- For weakly alternating compositions $\left(c_{i-1} \leq c_{i} \geq c_{i+1}\right), r \doteq .57614877$ by [3]. It follows from (5]) that $p_{m} \doteq 0.363144$ for $m \geq 25$.

Since the generating function for compositions has only a simple pole on its circle of convergence, the asymptotic estimate $A r^{-n}$ converges exponentially. Hence counting compositions can lead to a fairly accurate estimate for $r$. The value of $C$ is $1 / 2$ for unrestricted compositions and is 0.45636347 for Carlitz compositions [15]. C appears harder than $r$ to estimate by numerical methods.

Here is an alternative definition of gap-free based on the literature: A composition is gap-free if, whenever it contains two recurrent parts, say $a b$, it contains all recurrent parts between $a$ and $b$. This definition does not alter the conclusions of Theorem 17 d,e) because the fraction of compositions of $n$ that omit any particular recurrent subcompositions (such as the smallest recurrent part) is exponentially small. To see this, let $\mathcal{C}_{\Phi} \supset \mathcal{C}_{\Psi}$ be two families of compositions such that $\Psi$ excludes some recurrent subcompositions that can occur in $\mathcal{C}_{\Phi}$. It follows from Lemma $2(f)$ of [2] that $r_{\Phi}<r_{\Psi}$. (Recall that for each $\Phi$ we have radius $r$ determined by the equation $\lambda(r)=1$.)

Example 5 (Conjectures of Jaklič, Vitrih and Žagar) Let $\operatorname{Max}_{k}(n)\left(\right.$ resp. $\left.\operatorname{Min}_{k}(n)\right)$ denote the number of all compositions of $n$ such that there are more than $k$ copies of the maximal (resp. minimal) part. Jaklič et al. [5] conjectured that, when $k=1$

$$
\begin{aligned}
& \lim _{n \rightarrow \infty} \frac{\operatorname{Min}_{\mathrm{k}}(n+1)}{\operatorname{Min}_{\mathrm{k}}(n)}=2 \\
& \lim _{n \rightarrow \infty} \frac{\operatorname{Max}_{\mathrm{k}}(n+1)}{\operatorname{Max}_{\mathrm{k}}(n)}=2 .
\end{aligned}
$$

In fact, the conjectures hold for the compositions studied in this paper and all $k \geq 1$ provided 2 is replaced with $1 / r$ and Min is restricted to recurrent parts. The number of occurrences of any given recurrent part is $\Theta(n)$ for almost all recurrent locally restricted compositions of $n$ by [2]. Thus (6) follows immediately from the fact that the number of compositions of $n$ is asymptotic to $A r^{-n}$. We now prove (7). Note that

$$
\operatorname{Max}_{k}(n) \sim A r^{-n}\left(1-\sum_{i \leq k} g_{n}(i)\right) .
$$


By Theorem 1 f $), g_{n}(i) \sim g_{n+1}(i)$ and $g_{n}(i)$ is bounded away from zero as $n \rightarrow \infty$. Thus

$$
\frac{\operatorname{Max}_{\mathrm{k}}(n+1)}{\operatorname{Max}_{\mathrm{k}}(n)} \sim \frac{\left(1-\sum_{i \leq k} g_{n+1}(i)\right) A r^{-n-1}}{\left(1-\sum_{i \leq k} g_{n}(i)\right) A r^{-n}} \sim \frac{1-\sum_{i \leq k} g_{n+1}(i)}{1-\sum_{i \leq k} g_{n}(i)} \frac{1}{r} \sim \frac{1}{r} .
$$

One can change the definition of $\operatorname{Max}_{k}$ to mean exactly $k$ copies of the maximal part and a similar proof will hold for the revised (7).

Example 6 (Counterexamples without freeness) It was shown in Theorem 1(f) of [1] that when differences of adjacent parts are restricted to a finite set, the largest part is asymptotically almost surely of order $\sqrt{\log n}$, so the bound in Theorem 1 (a) fails.

\section{Individual Large Parts and Geometric Probability}

The following three lemmas are used in our proof of Theorem 1.

Lemma 1 (Large part separation) Let $\mathcal{C}$ be a class of $t$-free compositions. Suppose $\delta>0$ and $d>0$. Then there is a $P=P(\delta, d)$ and $N=N(\delta, d)$ such that the following holds. Choose a composition of $n$ at random from those having a part exceeding $P$. Choose a part $c_{i}$ at random from among those parts of size at least $P$. Then for all $n>N$ the probability that some part within d of $c_{i}$ exceeds $P-t$ is less than $\delta$.

Lemma 2 (Large subcompositions) Let $\mathcal{C}$ be a class of t-free, locally restricted compositions, let $\overrightarrow{\mathbf{s}}$ be a recurrent subcomposition of some $\overrightarrow{\mathbf{c}} \in \mathcal{C}$. The following are true.

(a) Let $X_{n}$ be the number of occurrences of $\overrightarrow{\mathbf{s}}$ in a random composition of $n$. The distribution of $X_{n}$ is asymptotically normal with mean and variance asymptotically proportional to $n$.

(b) There is a constant $B$ dependent only on $\mathcal{C}$ such that the probability that a random composition contains at least one copy of $\overrightarrow{\mathbf{s}}$ is at most Bnr ${ }^{\Sigma(\overrightarrow{\mathbf{s}})}$.

The following lemma proves most of Theorem 1 (a).

Lemma 3 (Geometric probabilities) We use the notation of Theorem 1 a).

(a) Equations (2) and (3) are true.

(b) Recall that $u_{k}$ is the limit (on $n$ ) of the ratio $\mathrm{E}\left(X_{k}(n)\right) / \mathrm{E}\left(X_{0}(n)\right)$. For all sufficiently large parts $k$ and $\ell$ depending on $\delta>0$, we have

$$
\left|\frac{u_{k} r^{-k}}{u_{\ell} r^{-\ell}}-1\right|<\delta .
$$

(c) We have $u_{k} \sim B r^{k}$ for some positive constant $B$. 


\section{Multiple Large Parts and Poisson Probability}

In this section we study sequences of large parts and obtain a joint Poisson distribution asymptotically. We remind the reader that logarithms are to the base $1 / r$.

A subcomposition of a composition is marked if the elements of the subsequence are distinguished in some manner. For example, in the composition 1,2,1,3,2 there is no marked subsequence 1,3 whereas $\dot{1}, 2,1, \dot{3}, 2$ and $1,2, \dot{1}, \dot{3}, 2$ each contain the marked subsequence $\dot{1}, \dot{3}$.

Lemma 4 Fix $k$ and a class $\mathcal{C}$ of t-free compositions. Let $A$ be such that the number of compositions of $n$ is asymptotic to $A r^{-n}$ and let $C$ be as in Theorem 1 . If $L_{1}, \ldots, L_{k}$ is a sequence of integers dependent on $n$ with

$$
\max \left(L_{i}\right)=o(n) \text { and } \min \left(L_{i}\right) \rightarrow \infty \text { as } n \rightarrow \infty,
$$

then the number of compositions $\overrightarrow{\mathbf{c}}$ of $n$ having $\overrightarrow{\mathbf{L}}=L_{1} \cdots L_{k}$ as a marked subsequence is

$$
(A+o(1)) \frac{(C n)^{k} r^{s-n}}{k !} \text { as } n \rightarrow \infty, \text { where } s=L_{1}+\cdots+L_{k} .
$$

We use the notation

$$
[m]_{k}:=m(m-1) \cdots(m-k+1) .
$$

The following result is Lemma 12 of [6], with minor changes in the statement.

Lemma 5 Suppose that $\zeta_{1}, \ldots, \zeta_{n}=\zeta_{1}(n), \ldots, \zeta_{n}(n)$ is a set of non-negative integer variables on a probability space $\Lambda_{n}, n=1,2, \ldots$, and there is a sequence of positive reals $\gamma(n)$ and constants $0<\alpha<$ 1 and $0<c<1$ such that

(i) $\gamma(n) \rightarrow \infty$ and $n-\gamma(n) \rightarrow \infty$;

(ii) for any fixed positive integers $\ell, m_{1}, \ldots, m_{\ell}$, and sequences $k_{i}(n)$ with $\left|k_{i}(n)-\gamma(n)\right|=O(1)$, for $1 \leq i \leq \ell$, we have

$$
\mathrm{E}\left(\left[\zeta_{k_{1}(n)}\right]_{m_{1}}\left[\zeta_{k_{2}(n)}\right]_{m_{2}} \cdots\left[\zeta_{k_{\ell}(n)}\right]_{m_{\ell}}\right) \sim \prod_{j=1}^{\ell} \alpha^{\left(k_{j}(n)-\gamma(n)\right) m_{j}}
$$

(iii) $\operatorname{Pr}\left(\zeta_{k(n)}>0\right)=O\left(c^{k(n)-\gamma(n)}\right)$ uniformly for all $k(n)>\gamma(n)$.

Then there exists a function $\omega(n) \rightarrow \infty$ so that for $k=\lfloor\gamma(n)-\omega(n)\rfloor$, the total variation distance between the distribution of $\left(\zeta_{k}, \zeta_{k+1}, \ldots, \zeta_{n}\right)$, and that of $\left(Z_{k}, Z_{k+1}, \ldots, Z_{n}\right)$ tends to 0 , where the $Z_{j}=Z_{j}(n)$ are independent Poisson random variables with $\mathrm{E} Z_{j}=\alpha^{j-\gamma(n)}$.

This is applied to obtain the following Poisson result for large parts, which we used with Mellin transforms to prove Theorem 1(b-d) and, with a result of Hitczenko and Knopfmacher [8] on sequences of geometric i.i.d. random variables, to prove Theorem 1(e,f).

Theorem 2 Let $\zeta_{j}$ be the number of parts of size $j$ in a random composition in $\mathcal{C}$ of size $n$. Let $\omega(n)$ be a function which goes to $\infty$ sufficiently slowly as in Lemma 5 and let $k=\lfloor\log (C n)-\omega(n)\rfloor$, where $C$ is the constant in Lemma 4 Then $\left\{\zeta_{j}: n \geq j \geq k\right\}$ are asymptotically independent Poisson random variables with mean $\mu_{j}=C n r^{j}$. 


\section{References}

[1] E.A. Bender and E.R. Canfield, Locally restricted compositions I. Restricted adjacent differences, Elec. J. Combin. 12(1) (2005) R57, 27pp.

[2] E.A. Bender and E.R. Canfield, Locally restricted compositions II. General restrictions and infinite matrices, Elec. J. Combin. 16 (2009) R108, 35pp.

[3] E.A. Bender and E.R. Canfield, Locally restricted compositions III. Adjacent-part periodic inequalities, Elec. J. Combin. 17 (2010) R145 9pp.

[4] P. Flajolet and R. Sedgewick, Analytic Combinatorics, Cambridge University Press, 2009.

[5] G. Jaklič, V. Vitrih and E. Žagar, Closed form formula for the number of restricted compositions, Bull. Aust. Math. Soc. 81 (2010) 289-297.

[6] Z.C. Gao and N.C. Wormald, The distribution of the maximum vertex degree in random planar maps, J. Combin. Theory, Ser. A 89 (2000) 201-230.

[7] W.M.Y. Goh and P. Hitczenko, Average number of distinct part sizes in a random Carlitz composition, Europ. J. Combinat. 23 (2002) 647-657.

[8] P. Hitczenko and A. Knopfmacher, Gap-free compositions and gap-free samples of geometric random variables, Discrete Math. 294 (2005) 225-239.

[9] P. Hitczenko and G. Louchard, Distinctness of compositions of an integer: A probablistic analysis, Random Structures and Algorithms 19 (2001) 407-437.

[10] P. Hitczenko and G. Stengle, Expected number of distinct part sizes in a random integer composition, Combinat. Probab. Comput. 9 (2000) 519-527.

[11] H.-K. Hwang and Y. Yeh, Measures of distinctness for random partitions and compositions of an integer, Adv. Appl. Math. 19 (1999) 378-414.

[12] T. Kato, Perturbation Theory for Linear Operators, Springer (1980).

[13] A. Knopfmacher and H. Prodinger, On Carlitz compositions, Europ. J. Combinat. 19 (1998) 579589.

[14] G. Louchard, The number of distinct part sizes of some multiplicity in compositions of an integer. A probabilistic analysis, Discrete random walks (Paris, 2003) 155-170 (electronic). Discrete Math. Theor. Comput. Sci. Proc., AC, Assoc. Discrete Math. Theor. Comput. Sci., Nancy, 2003.

[15] G. Louchard and H. Prodinger, Probabilistic analysis of Carlitz compositions, Discrete Math. and Theoret. Comput. Sci. 5 (2002) 71-96.

[16] F.W.J. Olver, Asymptotics and Special Functions, Academic Press, New York, 1974. 
